\title{
Image of the month: Abdominal aorta aneurysm rupture in the peritoneal cavity with a two-point aortocaval fistula formation
}

\author{
Authors: Olga Nikolaidou, ${ }^{A}$ Vasileios Kamperidis, ${ }^{B}$ Reggina Goulimari, ${ }^{C}$ Liana Panagiotidou ${ }^{C}$ and \\ Symeon Dimitriadis ${ }^{\mathrm{D}}$
}

\begin{abstract}
Abdominal aorta aneurysm rupture is a potential lethal complication and is surgical emergency. In addition, formation of a portal vein and abdominal aorta fistula is a possible, very rare complication that enforces complicated surgical management. Multidetector computed tomography with bolus injection tracking and multiplanar reconstruction is the modality of choice in revealing an aortic rupture and its potential complications.
\end{abstract}

KEYWORDS: abdominal aortic rupture, aortocaval fistula formation, computed tomography

DOI: $10.7861 /$ clinmed.2020-0943

\section{Case presentation}

A 76-year-old man was admitted to the emergency department with persistent, flank abdominal pain of sudden onset, radiated to the back. The patient had a history of arterial hypertension and an unremarkable family history. For the last 3 months, he was complaining about symptoms related to limp ischaemia.

Clinical examination revealed a low blood pressure of 73/40 $\mathrm{mmHg}$; tachycardia at a rate of 126 beats per minute; a palpable, pulsatile abdominal mass in the epigastrium; and low, near vanished, femoral pulses in both lower limbs. No pedal oedema was noticed. The patient was diaphoretic.

The patient underwent a chest-abdomen computed tomography angiography that revealed an extensive infrarenal abdominal aortic aneurysm, $9.3 \times 11.4 \mathrm{~cm}$, with signs of rupture into the peritoneal cavity and the retroperitoneum (Fig 1). Additionally, there was a two-point rupture into the inferior vena cava (IVC) resulting in aortocaval fistulas, manifested as beak signs (Fig 2). IVC and both common iliac veins showed the same

Authors: A consultant radiologist, George Papanikolaou General Hospital, Thessaloniki, Greece; ${ }^{B}$ consultant cardiologist and senior academic fellow, AHEPA Hospital, Thessaloniki, Greece; ${ }^{C}$ consultant radiologist, General Hospital of Xanthi, Xanthi, Greece; ${ }^{D}$ consultant radiologist and head of department, General Hospital of Xanthi, Xanthi, Greece



Fig 1. Axial computed tomography during arterial phase showing the abdominal aortic aneurysm rupture in the peritoneal cavity and the retroperitoneum. There is obscuring and enlarging of the right psoas muscle (yellow arrows) and a two-point rupture into the inferior vena cava (red arrows) creating two individual fistulas between the abdominal aorta and the inferior vena cava.

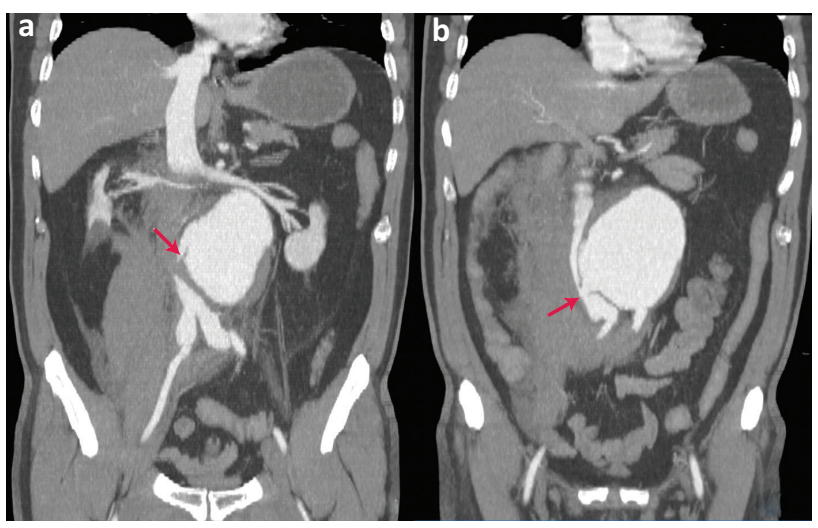

Fig 2. Coronal computed tomography showing the two-point rupture of the abdominal aortic aneurysm (arrows). The images accentuate the opacification of the entire inferior vena cava and common iliac veins lumen during the arterial phase, which is identical to the aortic lumen. 


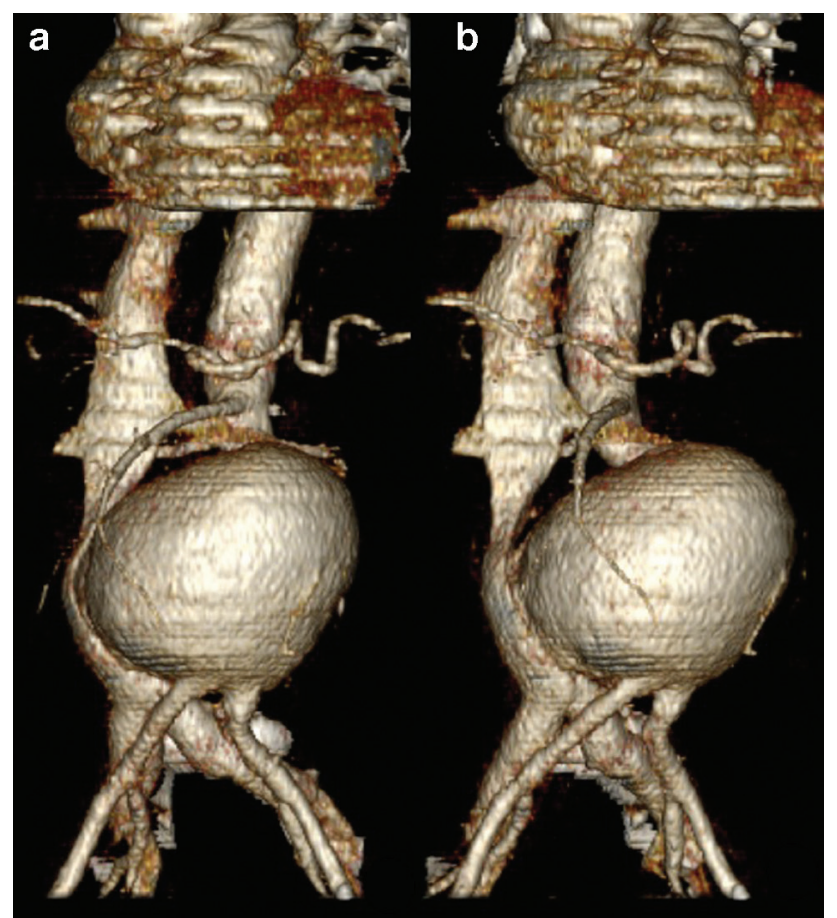

Fig 3. Volume rendered computed tomography showing the aortic aneurysm and the same opacification of the inferior vena cava as the abdominal aorta during the arterial phase. The enlarged lumen of the inferior vena cava and common iliac veins can be seen, due to high-pressure arterial blood inserted into the venous lumen.

opacification as the abdominal aorta during arterial phase along their entire course. Moreover, their calibres were enlarged due to the high pressure arterial blood inserted into the venous lumen from the aorta (Fig 3).
The patient was immediately transferred to a tertiary vascular surgery department, but unfortunately, he died upon the admission.

\section{Discussion}

Spontaneous rupture of an abdominal aortic aneurysm in the abdominal cavity accompanied by an aortocaval fistula is very rare $(<4 \%)$ and first described by Syme in $1831 .{ }^{1}$ Pathophysiologic mechanism suggests that it occurs due to chronic inflammatory reaction of the IVC wall from the increased tension that the abdominal aortic aneurysm exerts. The clinical presentation depends on the size, location and duration of the fistula formation and features a palpable pulsatile epigastric mass, tachycardia, classic 'water hammer' pulse and a pronounced bruit and thrill.' Treatment of choice includes a caval stent graft placement and open surgery on aortic rupture. Interventional approaches include an aorto-mono-iliac stent graft and a femoro-femoral crossover bypass. $^{2}$ -

\section{References}

1 Cinara IS, Davidovic LB, Kostic DM et al. Aorto-caval fistulas: a review of eighteen years experience. Acta Chir Belg 2005;105:616-20.

2 Siepe M, Koeppe S, Euringer W, Schlensak C. Aorto-caval fistula from acute rupture of an abdominal aortic aneurysm treated with a hybrid approach. J Vasc Surg 2009:49:1574-6.

Address for correspondence: Dr Olga Nikolaidou, Radiology Department, George Papanikolaou General Hospital, Papanikolaou Avenue, Thessaloniki 57010, Greece. Email: olganikolaidou@hotmail.com 